\title{
Modified energy harvesting figures of merit for stress- and strain-driven piezoelectric systems
}

\author{
James I. Roscow ${ }^{1, \text { a }}$, Holly Pearce ${ }^{1}$, Hamideh Khanbareh ${ }^{1}$, Sohini Kar-Narayan ${ }^{2}$, \\ and Chris R. Bowen ${ }^{1}$ \\ ${ }^{1}$ Materials and Structures Centre, Department of Mechanical Engineering, \\ University of Bath, Bath, UK \\ ${ }^{2}$ Department of Materials Science, University of Cambridge, Cambridge, UK
}

Received 4 September 2018 / Received in final form 17 January 2019 Published online 23 August 2019

\begin{abstract}
Piezoelectrics are an important class of materials for mechanical energy harvesting technologies. In this paper we evaluate the piezoelectric harvesting process and define the key material properties that should be considered for effective material design and selection. Porous piezoceramics have been shown previously to display improved harvesting properties compared to their dense counterparts due to the reduction in permittivity associated with the introduction of porosity. We further this concept by considering the effect of the increased mechanical compliance of porous piezoceramics on the energy conversion efficiency and output electrical power. Finite element modelling is used to investigate the effect of porosity on relevant energy harvesting figures of merit. The increase in compliance due to porosity is shown to increase both the amount of mechanical energy transmitted into the system under stress-driven conditions, and the stress-driven figure of merit, $\mathrm{FoM}_{33}^{X}$, despite a reduction in the electromechanical coupling coefficient. We show the importance of understanding whether a piezoelectric energy harvester is stress- or strain-driven, and demonstrate how porosity can be used to tailor the electrical and mechanical properties of piezoceramic harvesters. Finally, we derive two new figures of merit based on the consideration of each stage in the piezoelectric harvesting process and whether the system is stress- $\left(F_{i j}^{X}\right)$, or strain-driven $\left(F_{i j}^{x}\right)$.
\end{abstract}

\section{Introduction}

Piezoelectric energy harvesting is an important technology for converting ambient mechanical energy, a necessary by-product of thermodynamic processes, into useful electrical energy for powering wireless sensor networks, for example [1]. A variety of figures of merit have been derived to aid in the selection of materials for devices and provide insight into how piezoelectric materials can be engineered to enhance their energy harvesting properties. One frequently used merit index is

a e-mail: j.i.roscow@bath.ac.uk 
the electromechanical coupling coefficient, which is a measure of the efficiency of conversion of input mechanical energy into stored electrical energy, and vice versa:

$$
k^{2}=\frac{\text { Stored electrical energy }}{\text { Input mechanical energy }}=\frac{\text { Stored mechanical energy }}{\text { Input electrical energy }}
$$

and in terms of material properties, the coupling coefficient is given by:

$$
k_{i j}^{2}=\frac{d_{i j}^{2}}{\varepsilon_{i i}^{X} s_{j j}^{E}}
$$

where $d_{i j}$ is the piezoelectric strain coefficient, $\varepsilon_{i i}^{X}$ is the permittivity at constant stress and $s_{j j}^{E}$ is the mechanical compliance of the material under constant electric field conditions. A high coupling factor is not necessarily an indicator of the energy harvesting proficiency of a material, but rather relates to the efficiency of conversion of the input mechanical/electrical energy to stored electrical/mechanical energy. Piezoelectric energy harvesters, on the other hand, need to convert mechanical energy into useable, or output, electrical energy.

As recently pointed out by Uchino [2] and Deutz et al. [3], the electromechanical coupling coefficient is not the primary factor for predicting a piezoelectric's energy harvesting capabilities; instead the energy harvesting figure of merit derived by Islam and Priya, based on an applied stress, is commonly used [4]:

$$
\operatorname{FoM}_{i j}^{X}=\frac{d_{i j}^{2}}{\varepsilon_{i i}^{X}}
$$

The difference between the two merit indices in equations (2) and (3) is the lack of a compliance term in equation (3), which has been postulated to be a potential benefit for polymer-based piezoelectrics that exhibit high $\operatorname{FoM}_{i j}^{X}$ values, despite their low coupling coefficients, as a result of their high compliance and low piezoelectric coefficients $\left(d_{i j}\right)$ [3]. This concept is also relevant to the use of porosity to enhance the performance of piezoelectric materials, whereby pores are intentionally introduced into a piezoceramic in order to reduce the effective permittivity, whilst maintaining relatively high piezoelectric strain coefficients. This leads to increased longitudinal harvesting figures of merit, $\mathrm{FoM}_{33}^{X}$, with increasing porosity $[5,6]$.

The elastic compliance, which is used to determine $k^{2}$, is difficult to measure for porous piezoceramics as quality factors $\left(Q_{m}\right)$ decrease compared to dense piezoceramics [7]. This makes it challenging to accurately analyse the resonance data of porous piezoelectrics, which is required to obtain mechanical properties, such as stiffness or compliance, hence they are seldom reported in experimental studies. However, the decrease in stiffness of ceramic materials with increasing pore fraction is well known [8]. A decrease in the coupling coefficient with increasing porosity is therefore likely according to equation (2), which will be demonstrated using a finite element model later in this paper. Two questions arise from inspection of these two contrasting figures of merit with regards to the observed beneficial properties of porous piezoelectric energy harvesters. Firstly, does the introduction of porosity into dense piezoceramics reduce their efficiency as transducers of mechanical to electrical energy? Secondly, is efficiency the most important parameter if maximising the output power for a given vibrational energy source is the most important criteria? This paper will aim to explore these questions by analysis of the piezoelectric energy harvesting process with reference to relevant figures of merit and material properties in Section 2, before testing out the findings with data obtained from finite element analysis of porous piezoelectric materials in Section 3, followed by a discussion in Section 4 . 


\section{Derivation and analysis of figures of merit}

The output electrical energy extracted from a piezoelectric energy harvesting material depends on (i) the amount of mechanical energy applied to and transferred into the material, (ii) the efficiency of conversion of input mechanical energy to stored electrical energy and (iii) the efficiency of conversion of stored electrical energy into usable, or output, electrical energy. In this section, these three stages of piezoelectric energy harvesting will be analysed step-by-step to understand the origin of both the coupling factor in equation (2) and energy harvesting figure of merit due to an applied stress in equation (3), as well as considering the relevant merit indices for harvesting from an applied strain.

\subsection{Input mechanical energy (maximising $u_{\text {mech,in }}$ )}

Firstly, let us consider the application of mechanical energy, in the form of either a stress or a strain, to a piezoelectric material. If we mechanically excite the material, some of the input energy will be converted into stored mechanical energy; the amount of energy transferred from energy source into the harvester depends on the ratio of the mechanical impedances of each component [2]. For simplicity, we assume a linear relationship between stress $(X)$ and strain $(x)$, such that the material obeys Hooke's law:

$$
u_{\text {mech,in }}=\frac{1}{2} x X
$$

The stored mechanical energy per unit volume $\left(u_{\text {mech,in }}\right)$ can be calculated for an applied stress or strain, where stress and strain cases are denoted by superscripts $X$ and $x$ in the following equations, respectively. If we know the Young's modulus $(Y=X / x)$ of the material:

$$
\begin{aligned}
& u_{\text {mech,in }}^{X}=\frac{X^{2}}{2 Y} \\
& u_{\text {mech,in }}^{x}=\frac{x^{2} Y}{2} .
\end{aligned}
$$

For the applied stress case in equation (5), the stored mechanical energy is proportional to the inverse of the stiffness of the material, i.e. $u_{\text {mech,in }} \propto 1 / Y$, whereas for the applied strain case we observe that $u_{\text {mech,in }} \propto Y$. The effect of stiffness on the stored mechanical energy is shown in Figure 1, where it can be seen that for an applied stress (Condition 1 in Fig. 1) the stored mechanical energy is higher for the low stiffness material compared to the higher stiffness material. In the context of utilising porosity to enhance energy harvesting performance of a piezoceramic, this constitutes another potential benefit alongside the decrease in effective permittivity, which is often the primary motivation for selecting a porous piezoceramic [5]. The same principle applies to piezoelectric polymers, which typically have stiffnesses an order of magnitude below that of piezoelectric ceramics [9]. The converse is true if we consider an applied strain, as in Condition 2 in Figure 1, in which the material with higher stiffness stores more mechanical energy. In this case, a high stiffness material would also be favourable to achieve a high electromechanical coupling coefficient, $k^{2}$, see equation (2), which would be beneficial to efficiently convert the stored mechanical energy to stored electrical energy, thus yielding more usable electrical energy. 


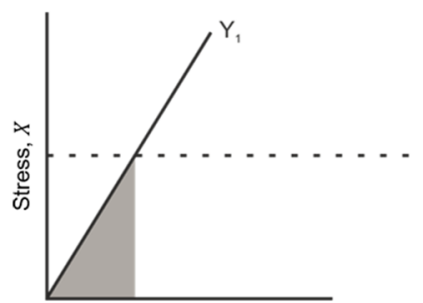

Strain, $x$

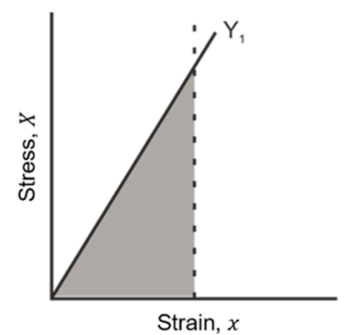

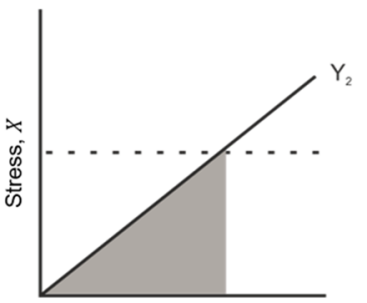

Strain, $x$

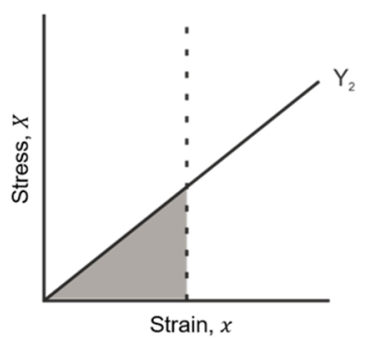

Condition 1 - constant stress

$Y_{1}>Y_{2}$

$u_{m e c h, 1}^{X}<u_{m e c h, 2}^{X}$

Condition 2 - constant strain

$Y_{1}>Y_{2}$

$u_{m e c h, 1}^{x}>u_{m e c h, 2}^{x}$

Fig. 1. Stress-strain curves demonstrating the difference in stored mechanical energy in materials with different stiffnesses for a constant stress (Condition 1) and a constant strain (Condition 2).

Throughout this paper, much of the discussion is presented in terms of mechanical compliance, $s$, which is the inverse of the Young's modulus, $Y$, such that $s=1 / Y$.

It is of benefit to consider applications where a harvester may need to operate at the two contrasting harvesting conditions of constant stress and constant strain. These can be related to the two modes of harvesting mechanical energy as discussed by Bowen et al. [1], namely inertial and kinematic energy harvesting; Crossley and Kar-Narayan then sub-divided kinematic harvesters into stress- or strain-driven systems [10]. For inertial harvesting, the active material is bonded to a mass that, when subject to an acceleration, induces a force (or stress) in the piezoelectric material, which is then converted into electrical energy; a harvester such as this is analogous to the applied stress case. In kinematic energy harvesting the active material is directly coupled to the relative motion of the mechanical energy source and could therefore be thought of as a strain-driven harvester; for example, harvesting strain energy from a car tyre. However, if a very high stiffness piezoelectric is bonded to a relatively compliant structure, such as the rubber of a car tyre, the efficiency of transmission of strain from the source into the active material would likely be poor. Hence, there is a requirement for mechanical impedance matching of the energy source and the piezoelectric element. Therefore, to define any given piezoelectric harvesting system as either purely stress- or strain-driven is difficult. The first part of our analysis has demonstrated that understanding the energy source is important before designing or selecting the appropriate harvesting material. Tuning the mechanical impedance of the piezoelectric to that of the source enables us to maximise the energy transferred into the harvesting material, giving us the optimum chance of obtaining a high output electrical power.

To summarise the discussion in this section, depending on whether we have a stress- or strain-driven system we can maximise the mechanical energy transferred into the material by:

(i) Stress-driven: select low stiffness, high compliance material.

(ii) Strain-driven: select high stiffness, low compliance material. 


\subsection{Conversion of input mechanical to stored mechanical and electrical energy (maximising $u_{\text {elec,stored) }}$}

The next stage in the analysis is to consider the conversion of input mechanical energy into stored electrical energy. The input mechanical energy, $u_{\text {mech,in }}$ is partially converted to stored electrical energy, $u_{\text {elec,stored }}$, via the piezoelectric effect, with some remaining as stored mechanical energy, $u_{\text {mech,stored }}$, and a small amount dissipated as heat (dielectric loss, $\tan \delta$ ). The amount of input mechanical energy that is converted to stored electrical energy per unit volume depends on the electromechanical coupling coefficient, $k^{2}[2]$ :

$$
u_{\text {elec,stored }}=k^{2} u_{\text {mech,in }}
$$

with the remaining stored mechanical energy given by:

$$
u_{\text {mech,stored }}=\left(1-k^{2}\right) u_{\text {mech,in }} \text {. }
$$

Clearly the stored electrical energy, $u_{\text {elec,stored }}$, for a given input mechanical energy, $u_{\text {mech,in, }}$ can be enhanced by improving the electromechanical coupling coefficient. However, a low conversion efficiency and low $k^{2}$ material may be suitable for a stressdriven harvester if the decrease in stiffness and increase in compliance results in an increase in $u_{\text {mech,in }}$ (Eq. (5)), such that it outweighs the accompanied decrease in $k^{2}$.

Piezoelectric energy harvesting figures of merit, such as equation (3), can be derived from the stored electrical energy due to mechanical excitation, as it is reasonable to assume that the output electrical energy is proportional to the stored electrical energy. At low, off- resonance frequencies $(\ll 100 \mathrm{~Hz})$ a piezoelectric material can be considered as a parallel plate capacitor [4]. The energy stored in a capacitor is:

$$
U_{\text {elec,stored }}=\frac{1}{2} C V^{2}
$$

where $C$ is the capacitance and $V$ is the piezoelectric voltage developed between the electrodes. We can relate the capacitance and voltage to the material properties. Firstly, the capacitance of a material is:

$$
C=\varepsilon_{0} \varepsilon_{33}^{X} \frac{A}{t}
$$

where $\varepsilon_{0}$ is the permittivity of free space $\left(\varepsilon_{0}=8.854 \times 10^{-12} \mathrm{~F} / \mathrm{m}\right), A$ is the crosssectional area and $t$ is the thickness of the material. The open circuit voltage generated due to the applied mechanical load depends on the piezoelectric strain coefficient, $d_{i j}$, and the constant stress permittivity, $\varepsilon_{i i}^{X}$; to simplify, we will consider a stress or strain applied in the 3-direction (i.e. the direction in which the material is poled). For an applied stress:

$$
V_{o c}=\frac{d_{33}}{\varepsilon_{33}^{X}} t X_{3}
$$

and for an applied strain:

$$
V_{o c}=\frac{d_{33}}{\varepsilon_{33}^{X}} t x_{3} Y .
$$


Substituting equations (10)-(12) into equation (9) leads to the stored electrical energy terms for both the stress and strain conditions ${ }^{1}$; we have divided by the volume of the material to calculate the specific energy terms, i.e. $u_{\text {elec,stored }}=U_{\text {elec,stored }} / A t$. For an applied stress:

$$
u_{\text {elec,stored }}=\frac{1}{2} \frac{d_{33}^{2}}{\varepsilon_{33}^{X}} X_{3}^{2}
$$

and for an applied strain:

$$
u_{\text {elec,stored }}=\frac{1}{2} \frac{d_{33}^{2}}{\varepsilon_{33}^{X}} x_{3}^{2} Y^{2} .
$$

For all piezoelectric harvesting materials and conditions, the stored electrical energy due to a mechanical input energy in the 3-direction is therefore proportional to $d_{33}^{2} / \varepsilon_{33}^{X}$, hence the interest in the constant stress energy harvesting figure of merit (Eq. (3)). Again, we see that the stiffness of the material has no effect in terms of the stored electrical energy due to an applied stress (Eq. (13)), whereas the stored electrical energy due to an applied strain is dependent on the square of the stiffness (Eq. (14)). This leads to a third figure of merit (written now in the general form rather than for the longitudinal direction) based on an applied strain as:

$$
\operatorname{FoM}_{i j}^{x}=\frac{d_{i j}^{2} Y^{2}}{\varepsilon_{i i}^{X}} .
$$

Rather than using the stiffness term, Rodig et al. defined this strain-driven figure of merit using both the open and closed circuit compliance, $s_{33}^{E}$ and $s_{33}^{D}$, respectively [11]:

$$
\operatorname{FoM}_{i j}^{x}=\frac{d_{i j}^{2}}{\varepsilon_{i i}^{X} s_{j j}^{E} s_{j j}^{D}} .
$$

The replacement of the single stiffness term in equation (15) with the open and closedcircuit compliance terms in equation (16) is more accurate in terms of the maximum electrical power output, as no power can be derived at either zero or infinite electrical impedance, i.e. short and open circuit conditions, respectively; in reality the output power is maximum between these two conditions $[3,10,11]$.

To summarise, the key points from this section are:

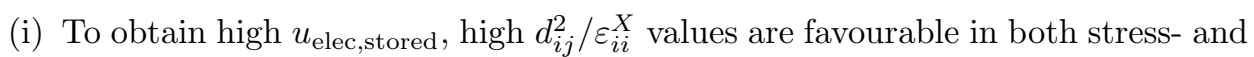
strain-driven harvesters.

(ii) In strain-driven harvesters, a low compliance is also beneficial to maximise $u_{\text {elec,stored }}$.

(iii) In stress-driven harvesters, the compliance of the material has no effect on $u_{\text {elec,stored }}$.

\subsection{Output electrical energy (maximising $\lambda_{\max }$ or $\lambda_{\max } / k^{2}$ )}

So far we have considered (i) the effect of stiffness on the conversion of applied mechanical energy to stored mechanical energy (Sect. 2.1) and (ii) the electrical

\footnotetext{
${ }^{1}$ These same equations can be derived by substituting equations (2), (5) and (6) into equation (7).
} 
energy stored by a piezoelectric due to an applied stress or strain (Sect. 2.2). To this point no electrical power has been extracted from the piezoelectric material. The final part of the harvesting process is the conversion of stored electrical to output electrical energy. The output electrical energy depends on the impedance of the external harvesting circuit, such that the electrical impedance of the material and the system should be matched to obtain maximum output power. The maximum output energy of a harvester due to a given stored mechanical energy depends on the transmission coefficient, $\lambda_{\max }[2,3]$, the full derivation of which can be found in [12]:

$$
\lambda_{\max }=\max \left(\frac{\text { output electrical energy }}{\text { input mechanical energy }}\right)=\max \left(\frac{u_{\text {elec,out }}}{u_{\text {mech,in }}}\right)
$$

where

$$
\lambda_{\max }=\left(\frac{1}{k}-\sqrt{\frac{1}{k^{2}}-1}\right)^{2} .
$$

The transmission coefficient enables the prediction of the maximum output electrical energy due to the input mechanical energy by rearranging equation (17):

$$
u_{\text {elec,out, } \max }=\lambda_{\max } u_{\text {mech,in }} .
$$

Substituting in equation (7) shows that the output electrical energy is proportional to the stored electrical energy (i.e. the energy harvesting figures of merit in Eq. (3) (applied stress) and (16) (applied strain)):

$$
u_{\text {elec,out } \max }=\frac{\lambda_{\max }}{k^{2}} u_{\text {elec,stored }}
$$

The maximum output electrical energy, $u_{\text {elec,out,max }}$, is shown in equation (20) to

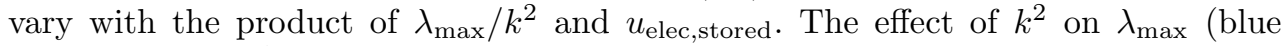
line) and $\lambda_{\max } / k^{2}$ (dashed green line) is shown in Figure 2. The relationship between between $k^{2}$ and $\lambda_{\max } / k^{2}$ varies linearly between $\lambda_{\max } / k^{2}=0.25$, when $k^{2}=0$, and $\lambda_{\max } / k^{2}=0.343$, when $k^{2}=0.5$. Therefore, even for small $k^{2}$ values, increases in the output electrical energy will always be achieved if $\mathrm{FoM}_{i j}^{X}$ or $\mathrm{FoM}_{i j}^{x}$ can be improved by at least 1.37 times (i.e. $0.343 / 0.25$ ), e.g. from the introduction of porosity, which may reduce $k^{2}$ due to an increase in compliance (Eq. (2)), but increase FoM $_{i j}^{X}$ due to a decrease in permittivity (Eq. (3)). Due to the dependence of the strain-driven figure of merit on the square of the stiffness of the material (Eq. (15)), porosity will not be an effective method to enhance $\mathrm{FoM}_{i j}^{x}$.

In this section, we have discussed what we consider to be the three main stages of piezoelectric energy harvesting: (i) transfer of mechanical energy into the piezoelectric material; (ii) conversion of stored mechanical energy into stored electrical energy due to the piezoelectric effect; and (iii) converting the stored electrical energy into useable electrical energy, i.e. the output electrical power. As discussed in Section 1, porosity has been shown previously to be a useful tool for tuning the properties of piezoelectric ceramics to enhance the energy harvesting capabilities $[5,6]$. The following section draws on the analysis to this point in the context of porous piezoelectrics for energy harvesting to understand how the relevant properties (piezoelectric coefficient, permittivity and compliance) vary due to the introduction of porosity, and depending on the mode of harvesting, i.e. whether it is stress- or strain-driven. 


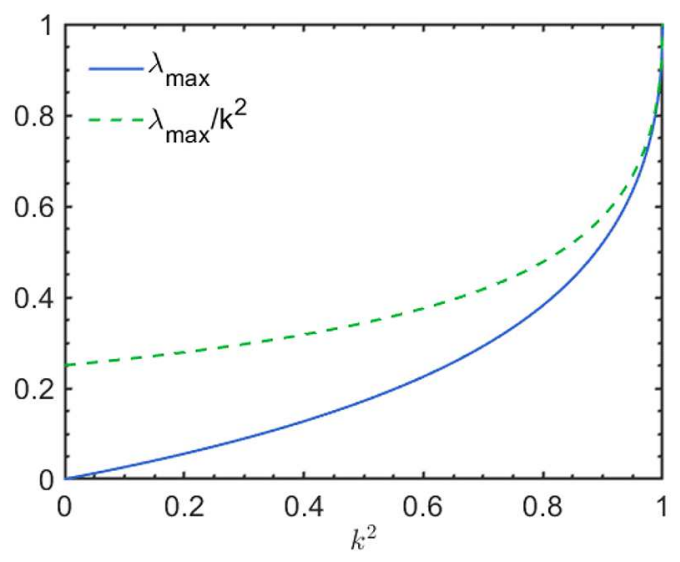

Fig. 2. Effect of $k^{2}$ on the transmission coefficient, $\lambda_{\max }$, and $\lambda_{\max } / k^{2}$ (both on $y$-axis).

To summarise, the key finding from this section is:

(i) A high $k^{2}$ is necessary for a high transmission coefficient, $\lambda_{\max }$, and $\lambda_{\max } / k^{2}$, however, when $k^{2}<0.5$, the main priority should be maximising $u_{\text {elec,stored }}$ since $\lambda_{\max } / k^{2}$ does not vary significantly in this range.

\section{Finite element model}

Finite element modelling has been used to provide a dataset to demonstrate the theory discussed in Section 2. The model presented is a porous poling model, used previously to explain the behaviour of porous lead zirconate titanate (PZT) materials with equiaxed pores [13], porous sandwich layer barium titanate [14] and porous barium titanate with aligned, anisotropic pores formed via the freeze casting method [6]. This model takes into account the complex electric field distribution during poling, which is the process required to align ferroelectric domains to yield a spontaneous polarisation. The spontaneous polarisation results in piezoelectric behaviour; when a mechanical stress or strain is applied to the material it results in a change in the net polarisation. This induces a piezoelectric voltage that can then be used to drive the flow of charge in an external circuit, thus enabling these materials to directly convert mechanical energy into electrical energy. From this model we can obtain material properties as a function of porosity, such as piezoelectric strain coefficients, $d_{i j}$, permittivity, $\varepsilon_{i i}^{X}$, mechanical compliances under both open and short circuit conditions, $s_{j j}^{E}$ and $s_{j j}^{D}$, respectively, and open circuit voltages due to applied stress and strain conditions. These effective properties have then been used to calculate electromechanical coupling coefficients, $k_{33}$ and $k_{33}^{2}$ (Eq. (2)), the energy harvesting figures of merit, $\mathrm{FoM}_{i j}^{X}$ and $\mathrm{FoM}_{i j}^{x}$ for constant stress and strain conditions (Eqs. (3) and (16)), respectively, and the transmission coefficient, $\lambda_{\max }$ (Eq. (19)). The stored mechanical and output electrical energy have been calculated using equations (5) and (6), and equation (19), respectively. Porous barium titanate with equiaxed, randomly distributed porosity will be the focus of this study, but the effect of porosity on both barium titanate and other ferroelectric ceramics, such as PZT, is comparable. Ansys APDL modelling software was used to conduct this study. 


\subsection{Modelling methodology}

To define a geometry with randomly distributed equiaxed porosity, a $5 \times 5 \times 5 \mathrm{~mm}$ cube was meshed into $30^{3}$ equiaxed elements. Elements were then randomly assigned the properties of either unpoled barium titanate [15] or air (relative permittivity, $\varepsilon_{r}=1$, zero stiffness) based on the desired pore fraction. A further step was taken to remove floating ceramic elements, i.e. those completely surrounded by air. The voltage degrees of freedom of the nodes at the surfaces of the block at $z=0$ and $z=l_{z}$ were coupled to simulate electrodes. A poling field was then applied parallel to the $z$-axis (calibrated by fitting to experimental data from [16] as detailed in $[13,14]$ ) to the model and the local electric field in each element analysed. This model has been validated against experimental data previously and shown to give a good representation of the behaviour of porous piezoceramics with equiaxed pores [13,14]. Where the local field in the z-direction in an element was greater than the coercive field of barium titanate $\left(E_{c}=0.5 \mathrm{kV} / \mathrm{mm}[15]\right)$ the properties of the element were changed from unpoled to poled barium titanate, with the primary polarisation direction parallel to the $z$-axis. After the distribution of poled material had been determined, an electric field was applied to the electrodes and the piezoelectric coefficients, $d_{33}$ and $d_{31}$, were calculated from the induced strain. The effective permittivity, $\varepsilon_{33}^{X}$, was calculated from the sum of the stored electrical energy using equations (9) and (10). Constant field compliance (short circuit conditions), $s_{33}^{E}$, was measured by applying a stress parallel to the $z$-direction and grounding the electrodes, i.e. defining the voltage at each electrode as zero, and using the strain to calculate the property using the relationship $s=x / X$. Similarly, $s_{11}^{E}$ was calculated from the strain in $x$ when a force was applied parallel to the $x$-axis, i.e. the 1-direction. The constant dielectric displacement (open circuit) compliance, $s_{j j}^{D}$, was measured in the same way as the short circuit compliance but with only one electrode grounded to $V=0 \mathrm{~V}$ and the other electrode free to vary in response to the piezoelectric effect. As the model geometries were generated randomly for each pore fraction, there was a slight spread in data depending on the distribution of porosity. The model was therefore run five times for each pore fraction.

\subsection{Finite element model results}

The effect of porosity on the effective piezoelectric strain coefficients, $d_{33}$ and $d_{31}$, is shown in Figure 3a, and the effect of porosity on the compliance is shown in Figure 3b. Introducing porosity into a ferroelectric ceramic leads to inhomogeneous poling field distributions that result in ferroelectric regions in the vicinity of pores remaining unpoled $[6,13,14]$, i.e. the net spontaneous polarisation after poling remains zero. These unpoled regions do not contribute to the piezoelectric response of the material, hence we see a decrease in the effective longitudinal and transverse piezoelectric coefficients with increasing pore fraction. The decrease in piezoelectric coefficients is detrimental to the harvesting properties (Eqs. (3) and (16)), however, this effect is offset by the larger decrease in the effective permittivity of the ferroelectric ceramic with increasing pore fraction, shown in Figure 3c. This leads to an increase in the longitudinal energy harvesting figure of merit due to an applied stress, $\mathrm{FoM}_{33}^{X}$, see Figure $4 \mathrm{a}$, as the permittivity falls at a faster rate than the longitudinal strain coefficient, which is the dominant factor in this case, see equation (3). However, the figure of merit $\operatorname{FoM}_{31}^{X}$ decreases with porosity due to significant decreases in $d_{31}$, see Figure 4a.

As discussed in Section 2, the stress-driven figure of merit is not dependent on the compliance of the material (Eq. (2)), however, for a strain-driven harvester, the figure of merit is inversely proportional to the product of the open and short circuit 
a

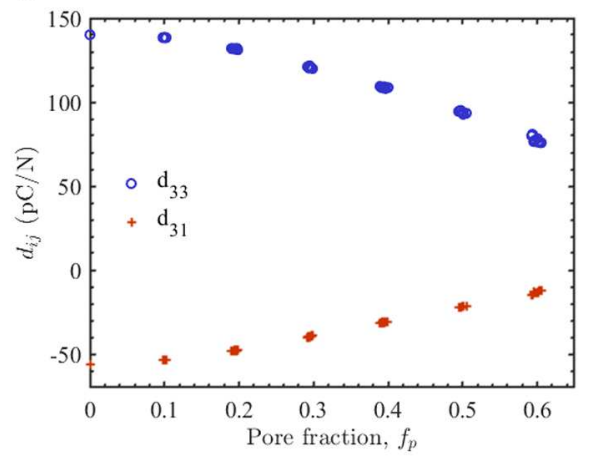

b

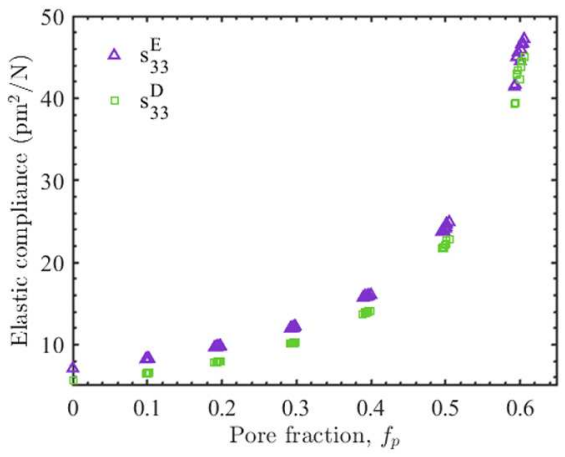

C

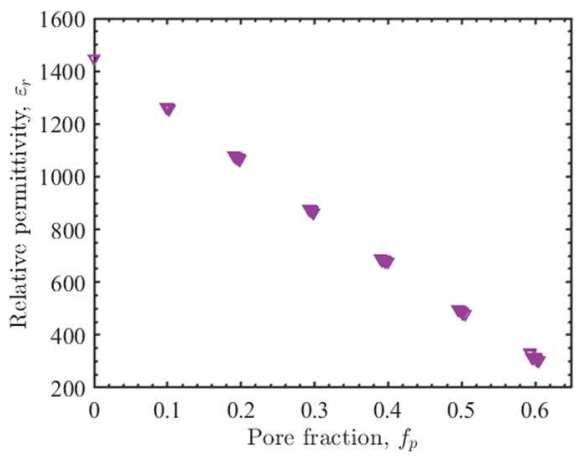

Fig. 3. Effect of pore fraction on (a) longitudinal $\left(d_{33}\right)$ and transverse $\left(d_{31}\right)$ piezoelectric strain coefficients, (b) constant field $\left(s_{33}^{E}\right)$ and constant dielectric displacement $\left(s_{33}^{D}\right)$ compliance and (c) relative permittivity $\left(\varepsilon_{r}=\varepsilon_{33}^{X} / \varepsilon_{0}\right.$, where $\varepsilon_{0}$ is the permittivity of free space $\left.\left(8.854 \times 10^{-12} \mathrm{~F} / \mathrm{m}\right)\right)$.

compliance (Eq. (16)), in addition to being proportional to $d_{i j}^{2} / \varepsilon_{i i}^{X}$. The longitudinal constant strain figure of merit, $\mathrm{FoM}_{33}^{x}$, for porous barium titanate is shown in Figure $4 \mathrm{~b}$ and decreases to close to zero for $f_{p}=0.6$ due to the significant increase in the compliance with increasing pore fraction, shown in Figure 3b. The transverse constant strain figure of merit, $\mathrm{FoM}_{31}^{x}$, also decreases with increasing pore fraction, reducing to almost zero at $f_{p}=0.4$, see Figure $4 \mathrm{~b}$. The longitudinal electromechanical coupling coefficient also decreases as a function of porosity, shown for both $k_{33}$ and $k_{33}^{2}$ in Figure 4c. Compliance increases at a similar rate to that which permittivity decreases, effectively cancelling these terms out, so that the associated drop in $d_{33}$ with porosity results in the reduction in $k_{33}^{2}$. The transmission coefficient, $\lambda_{\max }$, and $\lambda_{\max } / k^{2}$ are plotted as a function of porosity in Figure $4 \mathrm{~d}$; these two pre-factors are proportional to the maximum output electrical energy, see equations (19) and (20). The introduction of porosity significantly reduces $\lambda_{\max }$, which is related to a decrease in piezoelectric coefficients and increase in compliance, however, $\lambda_{\max } / k^{2}$ does not change significantly with increasing pore fraction, as predicted in Figure 2.

We can now use the data from the model to evaluate the discussion from Section 2. Firstly, using equations (4)-(6), the stored mechanical energy due to an applied stress and strain was calculated and is plotted as a function of porosity in Figures 5a and 5b, respectively. As predicted by equation (4), the stored mechanical energy increases with porosity in the applied stress case as the material becomes more compliant; the converse is true for the applied strain case where the dense and low compliance barium titanate stores the most mechanical energy. For the applied stress case, the 
a

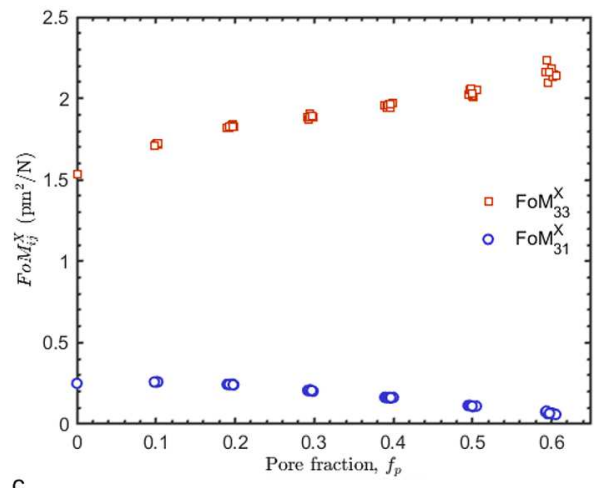

C

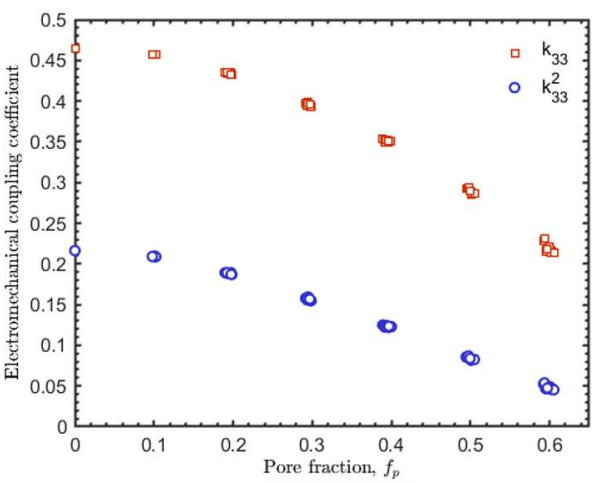

b

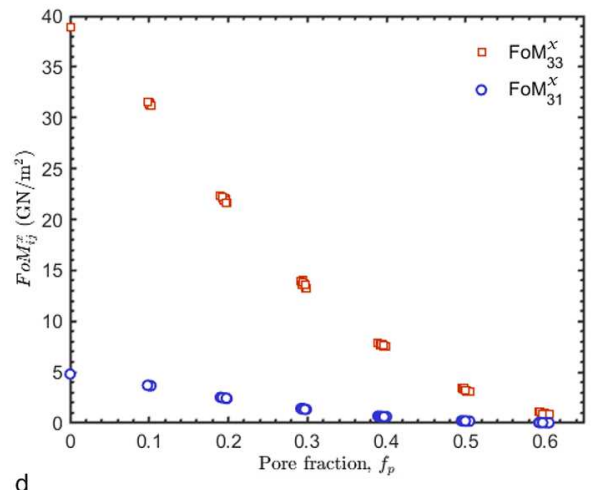

d

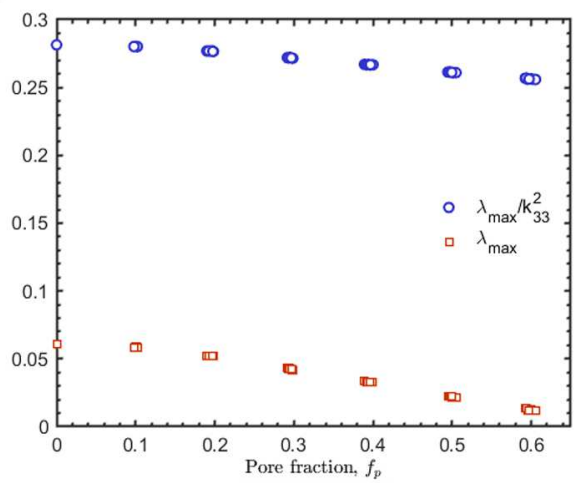

Fig. 4. Effect of pore fraction on the energy harvesting figures of merit for (a) constant stress $\left(\mathrm{FoM}_{i j}^{X}\right)$ and (b) constant strain, $\left(\mathrm{FoM}_{i j}^{x}\right) ;(\mathrm{c})$ longitudinal electromechanical coupling coefficient, $k_{33}$ and $k_{33}^{2}$, and (d) the transmission coefficient, $\lambda_{\max }$, and $\lambda_{\max } / k^{2}$, which are the respective pre-factors in equations (19) and (20).

barium titanate with the highest pore fraction examined of $f_{p}=0.6$ stores approximately six times more energy than the dense material; for the applied strain case, the stored energy decreases by six-fold for the material with $f_{p}=0.6$ compared to the dense material. The maximum output energy was calculated using equation (19), with the results shown in Figures $5 \mathrm{c}$ and $5 \mathrm{~d}$ for applied stress and applied strain cases, respectively. Unsurprisingly, the output energy due to an applied stress or an applied strain follow similar trends to that of the respective stress and strain-driven figures of merit shown in Figures $4 \mathrm{a}$ and $4 \mathrm{~b}$. This is predicted by equation (20) that tells us that $u_{\text {elec,out,max }}$ is proportional to both $\mathrm{FoM}_{33}^{X}$ and $\mathrm{FoM}_{33}^{x}$. The maximum output energy predicted by the model for harvesting energy from a mechanical stress occurs at $f_{p}=0.6$, which yields a $30 \%$ increase in output electrical energy compared to the dense barium titanate at $f_{p}=0$, see Figure $5 \mathrm{c}$.

The output open circuit voltages for the stress and strain cases were obtained from the model and shown in Figures $6 \mathrm{a}$ and $6 \mathrm{~b}$, respectively. Once more we see that for the given stress condition, the output voltage increases with increasing porosity as the voltage is related to the piezoelectric voltage coefficient, $g_{i j}=d_{i j} / \varepsilon_{33}^{X}$. The longitudinal voltage coefficient, $g_{33}$, increases with porosity as the permittivity, $\varepsilon_{33}^{X}$, reduces at a faster rate than the piezoelectric coefficient, $d_{33}$. The voltage due to a given strain decreases with porosity, however, as with all the strain cases in this section, the required input stress (or force) is significantly higher to achieve the same strain in both a low compliance dense material and a highly compliant porous 
a

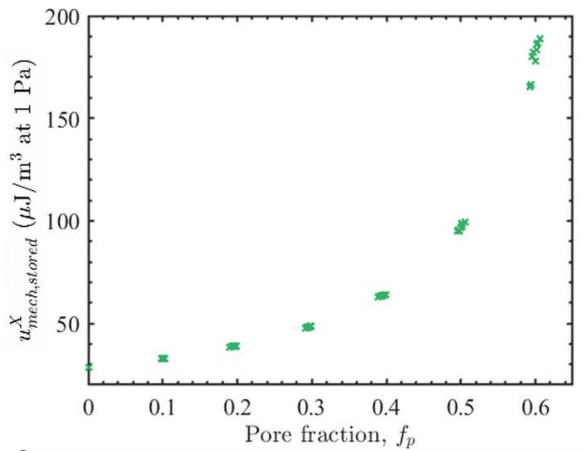

$c$

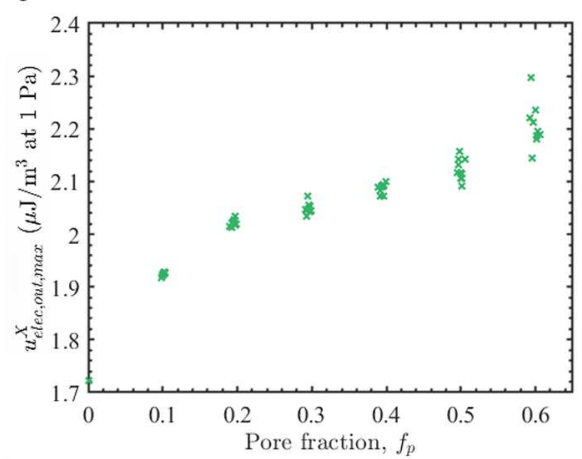

b

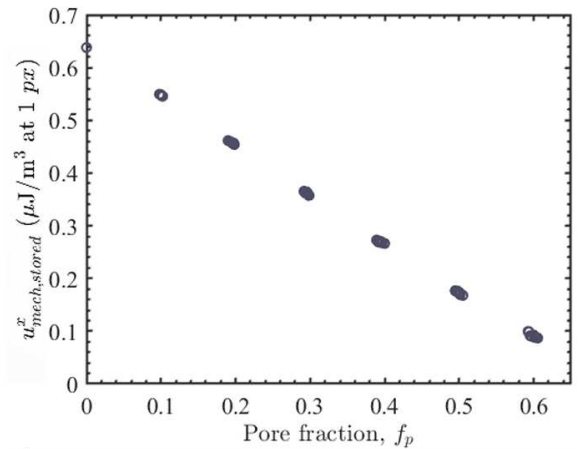

d

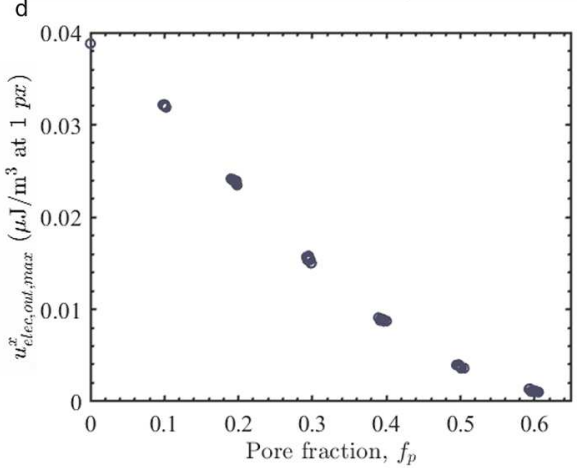

Fig. 5. Stored mechanical energy due to (a) an applied stress $\left(u_{\text {mech,stored }}^{X}\right)$ and (b) an applied strain ( $\left.u_{\text {mech,stored }}^{x}\right)$, and maximum output energy for (c) applied stress $\left(u_{\text {elec,out,max }}^{X}\right)$ and (d) strain ( $\left.u_{\text {elec,out,max }}^{x}\right)$, calculated from equation (19); 1 picostrain was used as applying $1 \mathrm{~Pa}$ to the dense material results in a strain on this order of magnitude. All data in these plots results from a force/strain applied longitudinally.
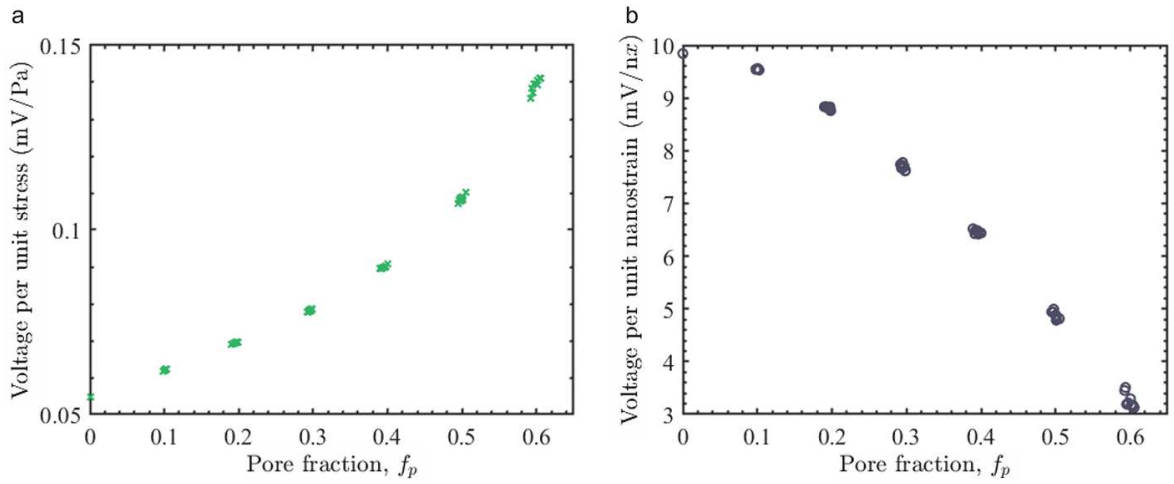

Fig. 6. Open circuit voltage response due to (a) an applied stress $(1 \mathrm{~Pa})$ and (b) and applied strain (1 nanostrain); the strain value was selected to give a similar voltage-axis scale.

material, which outweighs the fact the $g_{33}$ is lower for the dense barium titanate compared to the porous material. 


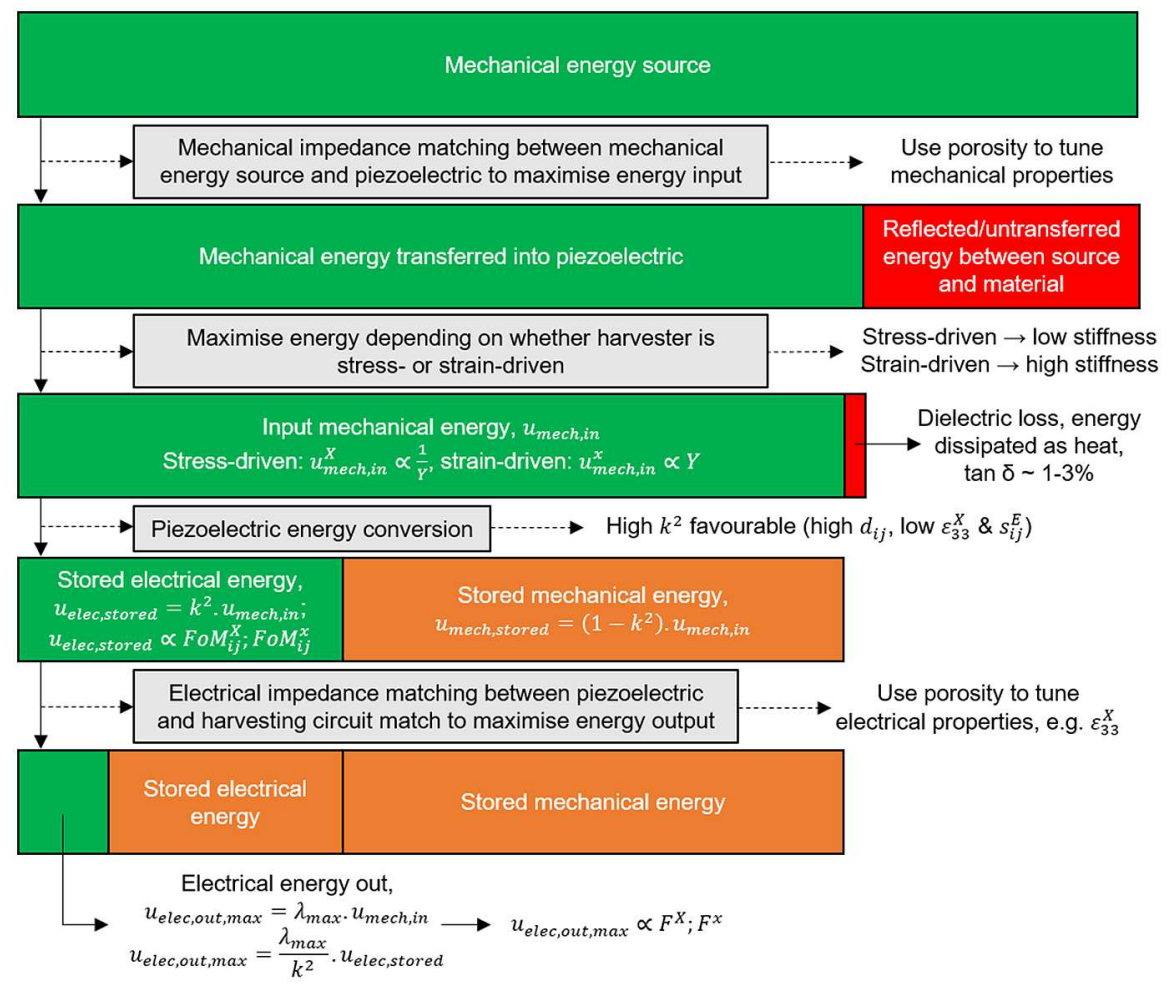

Fig. 7. Schematic showing piezoelectric material design considerations at each stage of vibrational energy harvesting process. Green boxes represent usable energy, orange boxes represent energy in the system that is not converted to usable electrical energy (although may be available for further harvesting cycles) and red boxes represent lost, i.e. non-recoverable energy.

\section{Discussion}

We have demonstrated through analysis of the various figures of merit (e.g. Eqs. (2), (3), (16) and (20)) for piezoelectric energy harvesting materials in Section 2 and a case study of the properties of porous piezoelectric ceramics in Section 3, the complexities around designing/selecting piezoelectric materials for energy harvesting applications. A summary of the energy harvesting process with reference to key material properties and equations derived in Section 2 is shown in Figure 7. The first stage considered in Figure 7 is the mechanical energy source, with only some of the energy transferred effectively into the material; the remaining energy is reflected, which depends on the mechanical impedance matching between the source and the material [2]. Next, a small amount of the mechanical energy is dissipated as heat due to extrinsic effects such as ferroelectric domain motion [17], i.e. dielectric loss, commonly given by the loss tangent, $\tan \delta$. The remaining stored mechanical energy is partially converted to stored electrical energy, with the unconverted mechanical energy available for subsequent cycles [3]. Of the stored electrical energy, which is proportional to the energy harvesting figures of merit discussed in Section 2 (Eqs. (3) and (16)), only some is converted to output electrical power.

Considering the complete piezoelectric energy harvesting process shown in Figure 7 and discussed in this paper, we can define two new figures of merit by substituting in the figures of merit derived from the electrical energy stored for both 

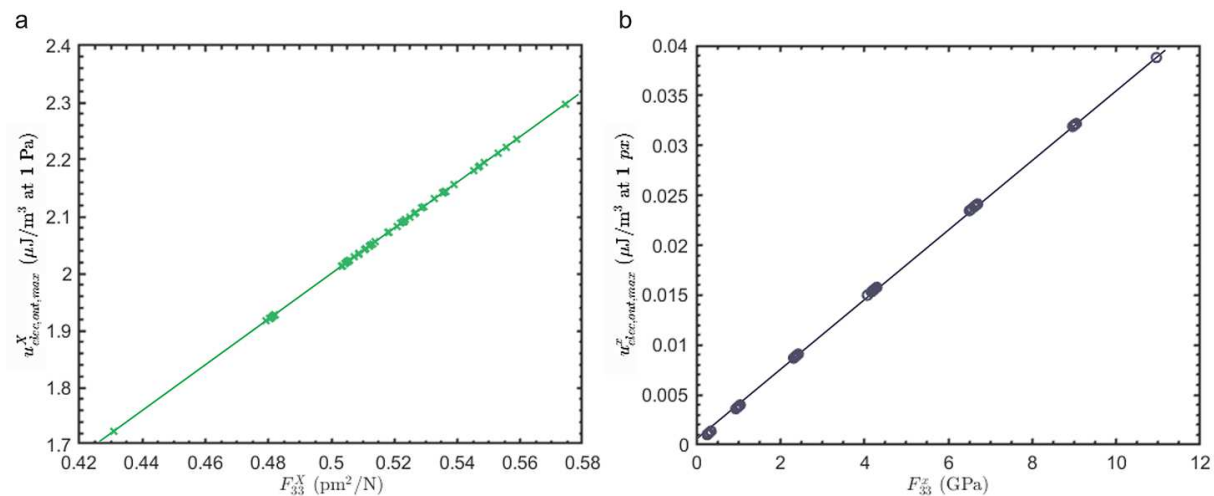

Fig. 8. The maximum output electrical energy for (a) stress- and (b) strain-driven porous barium titanate from Figure 7 plotted as a function of the newly derived figures of merit in longitudinal mode, $F_{33}^{X}$ (Eqs. (21) and (23)) and $F_{33}^{x}$ (Eqs. (22) and (24)), for stress and strain cases, respectively.

an applied stress (Eq. (3)) and strain (Eq. (16)) into equation (20) in Section 2.2. First, for an applied stress:

$$
F_{i j}^{X}=\frac{\lambda_{\max }}{k_{i j}^{2}} \frac{d_{i j}^{2}}{\varepsilon_{i i}^{X}}
$$

and for an applied strain:

$$
F_{i j}^{x}=\frac{\lambda_{\max }}{k_{i j}^{2}} \frac{d_{i j}^{2}}{\varepsilon_{i i}^{X} s_{j j}^{E} s_{j j}^{D}} .
$$

These can be written in terms of the electromechanical coupling coefficient, $k_{i j}^{2}$; for an applied stress:

$$
F_{i j}^{X}=\frac{\left(1-\sqrt{1-k_{i j}^{2}}\right)^{2} s_{j j}^{E}}{k_{i j}^{2}}
$$

and the strain case is:

$$
F_{i j}^{x}=\frac{\left(1-\sqrt{1-k_{i j}^{2}}\right)^{2}}{k_{i j}^{2} s_{j j}^{D}} .
$$

To demonstrate effectiveness of these new figures of merit we have plotted them against the modelled $u_{\text {elec,out,max }}$ from Section 3.2 in Figures $8 \mathrm{a}$ and $8 \mathrm{~b}$ for the stress and strain cases, respectively. We observe a linear fit between the longitudinal figures of merit, $F_{33}^{X}$ and $F_{33}^{x}$ and output energy, $u_{\text {elec,out,max }}$ in both cases, which demonstrates that the merit indices are successful in predicting the output electrical energy. One advantage of $F_{i j}^{X}$ and $F_{i j}^{x}$ is that they not only consider the stored electrical energy, such as in $\mathrm{FoM}_{i j}^{X}$ and $\mathrm{FoM}_{i j}^{x}$, but rather they consider the complete harvesting cycle shown in Figure 7 . These new figures of merit also clearly show the relevance of compliance to both the stress- and strain-driven cases. It is worth noting that the 
figures of merit for stored electrical energy in equations (3) and (16) are still relatively accurate in predicting the output power, and offer an easy ranking system as they do not require knowledge of a material's mechanical properties.

Depending on the type of energy harvesting system and characteristics of the input mechanical energy source, the mechanical properties of the piezoelectric can play an important role in maximising the harvesting capabilities of the material. For example, the input mechanical energy can be maximised by using either a more compliant material for a constant stress case, or by using a stiffer material for a constant strain case. A high electromechanical coupling coefficient is favourable in terms of yielding improved conversion of input mechanical energy to stored electrical energy and is therefore often discussed in terms of the efficiency with which a material can convert mechanical to electrical energy. In the stress-driven harvesting case, a lower electromechanical coupling coefficient due to a high compliance is outweighed by the fact mechanical energy is more effectively delivered into the material. Such a material may be considered to be 'less efficient' at converting mechanical into electrical energy (lower $k_{i j}^{2}$ ), despite delivering more output power for a given input mechanical stress. Nevertheless, for energy harvesting applications, such as for low power electronics, materials should be designed to yield maximum output power. As can be seen from the figures of merit discussed in this paper, e.g. equations (3), (16), (21) and (22), a high $d_{i j}^{2} / \varepsilon_{33}^{X}$ is required regardless of whether the system is stressor strain-driven.

With regards to the use of porous piezoelectrics for vibrational harvesting applications, we have shown that when the source is in the form of an applied stress, the increased compliance due to the introduction of pores that have effectively zero stiffness is not significantly detrimental to the overall performance, despite the accompanied reduction in $k_{i j}^{2}$ and $\lambda_{\max }$. In fact, when we consider that for optimum performance it is critical to match the mechanical impedances of the active material to the source energy, porosity provides an additional tool to engineer the properties of piezoelectric ceramics depending on the exact characteristics of the mechanical source energy (as well enabling independent tuning of piezoelectric coefficients and permittivity that has already been discussed in detail elsewhere $[5,6])$. Similarly, the electrical impedances of the piezoelectric and the harvesting circuit should also be matched, with porosity again providing a potential method to achieve this. Porous piezoceramic structures can also be infiltrated with polymers as a method to mitigate some of the detrimental effects of porosity on mechanical strength, for example. The introduction of porosity to match impedances between source and material has been of interest for many years for use of piezoelectric materials in low frequency SONAR devices [18-20] but has rarely been discussed for piezoelectric energy harvesting materials.

For harvesters subjected to very high stresses, the detrimental effect of porosity on the mechanical strength requires careful consideration [21], as do stress concentrations caused by the high pore fractions that may lead to depolarisation of the ferroelectric material, reducing the piezoelectric properties [22]. However, the largest mechanical stresses are likely to be present in strain-driven harvesters of high stiffness materials. For strain-driven harvesting, high stiffness materials are beneficial to maximise the input mechanical energy (Eq. (6)), electromechanical coupling and transmission coefficients (Eqs. (2) and (18), respectively) and the strain-driven figure of merit (Eq. (16)). The need for impedance matching is still critical as if, for arguments sake, we were to consider an infinitely stiff material, the harvesting-mode could no longer be considered as strain-driven as it would be impossible to transfer the strain into the material; for this to be case, the source must be significantly more stiff than the piezoelectric harvesting material. 


\subsection{Future directions in energy harvester design}

In this paper, we have provided an overview of the considerations that should be taken into account when designing or selecting a piezoelectric material for energy harvesting applications. Although written from the point of view of the materials engineer, to design an effective harvester it is evident the whole system needs to be considered. This means that a detailed understanding of the mechanical energy source and the application is as important as the ability to tune the properties of the active material. The individual fields of research on mechanical energy harvesting systems, piezoelectric energy harvesting materials (both in terms of specific material systems and composite/material structures), and harvesting electronics are sufficiently mature to see them start to be commercialised. The drive now should be to combine the best characteristics of the materials, harvester and electronics, with consideration of the other components and the complete system, to improve energy harvesting devices compared to the current state of the art.

We have shown that porosity can be an effective way of improving harvesting figures of merit in certain cases, namely in stress-driven systems. In reality, polymer second phases are likely to be necessary to provide the composite with sufficient mechanical toughness to survive the operating conditions, which can be relatively harsh in terms of stresses, strains and the lifetime of any material or device. Using polymeric matrices with piezoactive ceramics as the second phase may also be effective for stress-driven harvesters [3], overcoming the inherent brittleness of ceramic materials. However, whilst the effective permittivity remains inherently low for polymer-based composites, the piezoelectric strain coefficients are significantly lower than porous ceramic systems, which can adversely affect output electrical power.

The porous barium titanate microstructures modelled in Section 3 have equiaxed pores, such as those formed by methods such as the BURPS process. As often reported for porous ferroelectrics, an issue with these materials for use as energy harvesters are the poor transverse properties caused by significant reductions in the transverse piezoelectric strain coefficients, $d_{31}$. Perhaps the most common operation mode for piezoelectric energy harvesters is in bending, due to higher strain profiles, so this is a significant drawback for using porous or composite materials. However, commercially available macro-fibre composites (MFCs) with PZT-5A fibres have $d_{31}$ values close to that of the dense PZT $\left(d_{31}=-210 \mathrm{pC} / \mathrm{N}\right.$ for a P1-type MFC from Smart Material, USA) by virtue of the fibres being aligned in the 1-direction; as there is no connectivity in the 2 -direction, it is likely the $d_{32}$ coefficient is close to zero. Processing techniques for fabricating porous ceramics have advanced significantly in the past decade or so and therefore much more complex structures can be achieved with excellent control of microstructures possible, including long-range alignment of both ceramic and porous phases. Advanced processing methods of interest include freeze casting [6,23], self-organisational methods, such as gel casting [24] and ionotropic gelation $[25,26]$, and additive manufacturing, i.e. printing technologies [27]. Using these methods to produce 'pseudo-fibre composites' may prove advantageous for reducing the cost of commercially available MFCs by being able to produce them on a larger scale with excellent control over volume fractions of each phase, thus giving control over longitudinal and transverse piezoelectric coefficients (i.e. keeping them as high as possible), dielectric properties, and mechanical properties, for example. An additional step of sectioning the sintered bodies would likely be required in this case, although freeze casting offers the potential for producing near-net shape materials. This is an area of research that has yet to be explored in depth. 


\section{Conclusions}

A detailed overview of the complete piezoelectric energy harvesting process has been described to aid with effective materials selection and design. Whether the stiffness of the material has an effect on its harvesting ability depends on if a harvester is stress- or strain-driven. In a stress-driven system, selecting a low stiffness material, for example a porous piezoceramic, allows more efficient transfer of mechanical energy into the material, which can outweigh the decrease in electromechanical coupling and transmission coefficients. Electrical impedance matching between the piezoelectric material and the harvesting cicuit is also important to maximise the harvested energy.

In all cases the electrical output energy should be the key design consideration, regardless of whether that reduces the efficiency at which the piezoelectric material converts mechanical to electrical energy, i.e. the $k_{i j}^{2}$ of a piezoelectric. We have derived two new figures of merit, $F_{i j}^{X}$ and $F_{i j}^{x}$, for stress- and strain-driven piezoelectric harvesting materials, respectively. These merit indices take into account each step of the harvesting process and assess the material properties required to obtain a high output electrical energy, rather than stored electrical energy from which previous figures of merit have been derived $\left(\mathrm{FoM}_{i j}^{X}\right.$ and $\mathrm{FoM}_{i j}^{x}$ for stress and strain, respectively). In both stress- and strain-driven systems, a high $d_{i j}^{2} / \varepsilon_{i i}^{X}$ is required to achieve high output electrical power. For stress-driven systems, materials with high piezoelectric strain coefficients, $d_{i j}$, and low permittivity, $\varepsilon_{i i}^{X}$ are favourable, and increased compliance may be an additional benefit, making polymer, composite or porous piezoelectric materials promising. In strain-driven systems, the low permittivity of porous piezoceramics is outweighed by their increased compliance, which results in a decrease in both $F_{i j}^{x}$ and $\mathrm{FoM}_{i j}^{x}$ figures of merit. In these cases a high $d_{i j}$ coefficient and low compliance should be the main criteria used to select a material, however, mechanical impedance matching must be carefully considered.

SK-N is grateful for support from the European Research Council through an ERC Starting Grant (Grant no. ERC-2014-STG-639526, NANOGEN). CRB would like to acknowledge funding from the European Research Council under the European Union's Seventh Framework Programme (FP/2007-2013)/ERC Grant Agreement No. 320963 on Novel Energy Materials, Engineering Science and Integrated Systems (NEMESIS).

\section{Author contribution statement}

The concept for the paper was developed through discussions between all of the authors. JIR wrote the majority of the initial draft manuscript and conducted the modelling studies, with valuable input from all co-authors throughout.

Open Access This is an open access article distributed under the terms of the Creative Commons Attribution License (http://creativecommons.org/licenses/by/4.0), which permits unrestricted use, distribution, and reproduction in any medium, provided the original work is properly cited.

\section{References}

1. C.R. Bowen, H.A. Kim, P.M. Weaver, S. Dunn, Energy Environ. Sci. 7, 25 (2013)

2. K. Uchino, Energy Technol. 6, 829 (2018)

3. D.B. Deutz, J.-A. Pascoe, B. Schelen, S. van der Zwaag, D.M. de Leeuw, P. Groen, Mater. Horiz. 5, 444 (2018) 
4. R.A. Islam, S. Priya, Appl. Phys. Lett. 88, 032903 (2006)

5. J.I. Roscow, Y. Zhang, J. Taylor, C.R. Bowen, Eur. Phys. J. Special Topics 224, 2949 (2015)

6. J.I. Roscow, Y. Zhang, M.J. Kraśny, R.W.C. Lewis, J.T. Taylor, C.R. Bowen, J. Phys. D 51, 225301 (2018)

7. A.N. Rybyanets, IEEE Trans. Ultrason., Ferroelectr., Freq. Control 58, 1492 (2011)

8. A.P. Roberts, E.J. Garboczi, J. Am. Ceram. Soc. 83, 3041 (2000)

9. V.V. Varadan, Y.R. Roh, V.K. Varadan, R.H. Tancrell, in Proceedings, IEEE Ultrasonics Symposium (1989), p. 727

10. S. Crossley, S. Kar-Narayan, Nanotechnology 26, 344001 (2015)

11. T. Rödig, A. Schönecker, G. Gerlach, J. Am. Ceram. Soc. 93, 901 (2010)

12. K. Uchino, J.R. Giniewicz, in Micromechatronics (CRC Press, New York, 2003), p. 131

13. R.W.C. Lewis, A.C.E. Dent, R. Stevens, C.R. Bowen, Smart Mater. Struct. 20, 085002 (2011)

14. J.I. Roscow, R.W.C. Lewis, J. Taylor, C.R. Bowen, Acta Mater. 128, 207 (2017)

15. D. Berlincourt, H.A. Krueger, C. Near, Technical Publication TP-226, Morgan Electroceramics, 1999, pp. 1-12

16. J.I. Roscow, J. Taylor, C.R. Bowen, Ferroelectrics 498, 40 (2016)

17. A.J. Moulson, J.M. Herbert, in Electroceramics (Wiley, Chichester, 2003), p. 71

18. T. Arai, K. Ayusawa, H. Sato, T. Miyata, K. Kazutami, K. Keiichi, Jpn. J. Appl. Phys. 30, $2253(1991)$

19. S. Marselli, V. Pavia, C. Galassi, E. Roncari, F. Cranciun, G. Guidarelli, J. Acoust. Soc. Am. 106, 733 (1999)

20. H. Kara, R. Ramesh, R. Stevens, C.R. Bowen, IEEE Trans. Ultrason., Ferroelectr., Freq. Control 50, 289 (2003)

21. J. Seuba, S. Deville, C. Guizard, A.J. Stevenson, Sci. Rep. 6, 24326 (2016)

22. Y.A. Genenko, J. Glaum, M.J. Hoffmann, K. Albe, Mater. Sci. Eng. B 192, 52 (2015)

23. Y. Zhang, M. Xie, J. Roscow, Y. Bao, K. Zhou, D. Zhang, C.R. Bowen, J. Mater. Chem. A 5, 6569 (2017)

24. W. Liu, J. Xu, Y. Wang, H. Xu, X. Xi, J. Yang, J. Am. Ceram. Soc. 96, 1827 (2013)

25. W. Liu, N. Li, Y. Wang, H. Xu, J. Wang, J. Yang, J. Eur. Ceram. Soc. 35, 3467 (2015)

26. W. Liu, L. Lv, Y. Li, Y. Wang, J. Wang, C. Xue, Y. Dong, J. Yang, Ceram. Int. 43, $6542(2017)$

27. M. Smith, Y.S. Choi, C. Boughey, S. Kar-Narayan, Flex. Print. Electr. 2, 015004 (2017) 